\title{
Especie nueva de Scybalocanthon (Coleoptera: Scarabaeinae: Canthonini) y descripción de la variación del órgano genital masculino
}

\author{
A new species of Scybalocanthon (Coleoptera: Scarabaeinae: Canthonini) and description of the \\ variation of the male genitalia
}

\author{
Fredy Molano Rendón ${ }^{1 *}$ y Claudia Alejandra Medina Uribe ${ }^{2}$ \\ ${ }^{I}$ Grupo de Investigación Sistemática Biológica, Laboratorio de Entomología, Museo de Historia Natural "Luis Gonzalo Andrade”, Escuela de Ciencias \\ Biológicas, Universidad Pedagógica y Tecnológica de Colombia. Boyacá, Colombia. \\ ${ }^{2}$ Colecciones Biológicas, Instituto Alexander von Humboldt. Boyacá, Colombia. \\ *Correspondencia: fredymol@gmail.com
}

\begin{abstract}
Resumen. Se describe Scybalocanthon arcabuquensis Molano y Medina, sp.nov. La distribución de esta especie se encuentra restringida a los bosques de roble del municipio de Arcabuco, Departamento de Boyacá, sobre la cordillera Oriental de los Andes en Colombia. El órgano genital del macho se describe e ilustra, se discute la variación de las estructuras del saco interno de varias especies del género, y se proponen algunas estructuras del órgano genital como posibles caracteres únicos que definen el género.
\end{abstract}

Palabras clave: taxonomía, escarabajos coprófagos, América del Sur, Colombia.

\begin{abstract}
Scybalocanthon arcabuquensis Molano y Medina, sp. nov. is described. The species is restricted to the oak forest at Arcabuco municipality, department of Boyacá, Eastern Cordillera of the Colombian Andes. The male genitalia is described and illustrated, the variation of structures of the internal sac is discussed for various species of the genus and some structures from the internal male genitalia are proposed as possible unique characters useful for defining the genus.
\end{abstract}

Key words: taxonomy, dung beetles, South America, Colombia.

\section{Introducción}

Scybalocanthon fue descrito por Martínez (1948) de ejemplares de Costa Rica, antiguamente descritos como Canthon moniliatus. Aunque este autor argumenta que las descripciones de muchas especies son cortas y poco detalladas, como en este caso, encontró caracteres de peso suficientes para separar Canthon moniliatus en otro género. Según Martínez (1948), el principal carácter que separa Scybalocanthon de Canthon es: "el primer tarso de las patas medias y posteriores, que es notablemente más corto que el segundo; además, la forma de los tarsos de las mismas patas, la denticulación del borde externo de las tibias anteriores, el espolón de las mismas sin diferenciación para ambos sexos, la forma del labio". Más adelante, Martínez (1949) realizó una corrección en la descripción del género: "pigidio sin margen basal que lo separe del propigidio, proepisternos sin carena transversal ni con dentículo en el borde lateral". En esta última publicación, Martínez

Recibido: 29 septiembre 2009; aceptado: noviembre 2010 incluía a $S$. moniliatus y $S$. zischkai como únicas especies del género, aunque sugirió que Canthon cyanocephalus y C. trimaculatus deberían incluirse en Scybalocanthon. En los siguientes años se describieron nuevas especies de este género (Pereira y Martínez, 1956). En la actualidad se reconocen 16 especies, 13 de las cuales se reubicaron en Canthon: Scybalocanthon aereus (Schmidt, 1922); S. balachowskyi Martínez y Halffter, 1972; S. cyanocephalus (Harold, 1868); S. darlingtoni (Paulian, 1939); S. imitans (Harold, 1868); S. kastneri (Balthasar, 1939); S. kelleri Pereira y Martínez, 1956; S. maculatus (Schmidt, 1920); S. moniliatus (Bates, 1887); S. nigellus (Schmidt, 1922); S. nigriceps (Harold, 1868); S. pinopterus (Kirsch, 1873); S. pygidialis (Schmidt, 1922); S. sexspilotum (Guerin, 1855); S. trimaculatus (Schmidt, 1922) y S. zischkai Martínez, 1949.

Las especies de Scybalocanthon se distribuyen ampliamente en bosques bajos húmedos y secos en Centro y Sur América. La biología de algunas fue descrita por Martínez y Halffter (1972), quienes argumentan que la mayoría de las especies de este género son de actividad diurna, especialmente en las horas de mayor insolación; 
generalmente se recolectan en bosques, con trampas cebadas con excremento humano fresco. Estos autores observaron a $S$. balachowskyi con movimientos de vuelo ágil; haciendo pequeñas bolas de excremento, ocultas bajo las hojas caídas del bosque y enterrando a los pocos centímetros. Las especies de Scybalocanthon están asociadas con excremento de variados vertebrados, incluyendo monos aulladores, pero también se han encontrado en hongos y carroña, como se ha observado en S. moniliatus en la isla de Barro Colorado en Panamá (Howden y Young, 1981). Esta especie es abundante en la isla y se le ve activa durante el día, perchada sobre las hojas, esperando las defecaciones de monos aulladores (Alouatta palliata), también numerosos en Barro Colorado (C.A. Medina obs. per.).

Aunque la sistemática de Scybalocanthon y géneros relacionados como Canthon, Hansreia y Sylvicanthon no está definida, en el análisis filogenético realizado por Medina et al. (2003) las especies de este género incluidas en el análisis se agruparon en un clado. En la revisión de la morfología interna del macho de las especies de Scybalocanthon estudiadas en el presente trabajo se observaron estructuras que pueden ser caracteres únicos compartidos que ayudan a definir el género como un grupo monofilético.

\section{Materiales y métodos}

Se examinaron 310 ejemplares de las siguientes colecciones: Colección de Artrópodos del Instituto Alexander Von Humboldt, Villa de Leyva, Boyacá, Colombia (IAVH); Colección de Escarabajos Coprófagos de Colombia, Bogotá, Colombia (CECC); y Colección Entomológica del Museo de Historia Natural "Luis Gonzalo Andrade", Universidad Pedagógica y Tecnológica de Colombia, Tunja, Boyacá, Colombia (UPTC).

Se revisaron los tarsos medios y posteriores, el pigidio, el hipómero, el edéago y el saco interno del edéago de las siguientes especies: Scybalocanthon maculatus, $S$. nigriceps, $S$. aff. pygidialis $1, S$. aff. pygidialis $2, S$. aff. pygidialis 3, S. aff. pygidialis 4, S. trimaculatus, $S$. darlingtoni, S. kelleri, S. moniliatus, $S$. aereus y $S$. aff. nigellus. Para comparar tamaños y proporciones de las estructuras estudiadas se adaptó un acetato impreso con una cuadrícula de $0.1 \mathrm{~mm}$, el cual se sobrepuso sobre la estructura cuando fue necesario. Se ilustraron las estructuras de la morfología externa e interna así como los escleritos del saco interno del edéago y epifaringe, entre otras. La nomenclatura para las estructuras del órgano genital masculino es la propuesta por Medina et al. (2003), con algunas modificaciones; se incluye la región submedial (Fig. 15a), región apical (Fig. 15b) y esclerito basal (Fig. 15d).

Scybalocanthon arcabuquensis Molano y Medina, n. sp.

Diagnosis. Se diferencia de las demás especies por presentar un único patrón de mancha ancha verde oscuro en el centro del pronoto; élitros muy brillantes de color verde oscuro; hipómero con sutura en el borde interno evidente y termina en proyección corta (Fig. 8); por la forma de los parámeros del edéago (Fig. 14) y la forma de los escleritos del saco interno (Figs. 16-18).

Holotipo macho. Longitud total 7,5 mm. Cuerpo oval, lados paralelos (Fig. 1). Coloración verde oscuro a casi negro y brillante sobre la cabeza, parte central del pronoto, élitros, hipómero, mesoesterno, parte lateral del metaesterno y patas. Color amarillo (transparente) en la parte lateral del pronoto, procoxa, región medial del fémur medio y posterior, región central del metaesterno, abdomen y pigidio. Cabeza. Borde anterior bidentada, dientes poco agudos con muesca en $\mathrm{V}$; línea de setas en la parte basal de los dientes (Fig. 2). Sutura clipeal definida y poco profunda. Gena formando un arco pequeño, con dentículo justo después de la sutura clipeal. Ojo 1/3 la longitud total de la gena, sin marginación. Borde cefálico posterior con suave marginación. Superficie dorsal de la cabeza con punturación muy fina. Borde anterior de la cabeza en posición ventral con leve marginación y superficie plana con numerosas setas (Fig. 2). Borde anterior del mentón escotado en forma de U muy abierta (Fig. 3). Separación entre el submentón y la gula con suave arco y escasas setas. Palpo maxilar con primer artejo subcilíndrico, ancho apicalmente; segundo artejo ensanchado medialmente, con base y ápice de borde irregular, ligeramente más largo que el primero; tercer artejo subcuadrado y cuarto artejo ensanchado medialmente, subigual en tamaño a los tres anteriores juntos (Fig. 4). Palpo labial con primer artejo subcilíndrico; segundo artejo subcuadrado, con pequeña proyección en el borde interno, $1 / 2$ la longitud del primero; tercer artejo cilíndrico, mas estrecho que los anteriores (Fig. 5). Epifaringe con proceso medial que sobrepasa el borde anterior; setas en el borde anterior cortas y subiguales en tamaño; hilera de setas laterales decrecen en tamaño desde el borde anterior hacia la base; sutura transversal evidente; tormal subigual en tamaño a los brazos laterales (Fig. 6). Tórax. Pronoto transversal (Fig. 7); ángulos anteriores agudos dirigidos levemente hacia afuera. Borde lateral levemente angulado. Ángulo posterior con profunda saliente. Superficie del pronoto con puntuación muy fina, glabra y con diseño (Fig. 7). Hipómero no escavado, con quilla pequeña en borde interno y sin tubérculo en borde 
lateral (Fig. 8). Mesoesterno rugoso. Metaesterno plano con puntuación muy fina. Élitros: con 9 estrías poco evidentes, formadas por puntos como tubérculos separados $1 / 3$ entre sí , estría 8 sin tocar la base. Interestrías con puntuación muy fina casi imperceptibles y muy brillante. Patas: fémur anterior con quilla corta, un tercio la longitud total del fémur. Tibia anterior con 3 dientes en el borde externo, los 2 apicales mas próximos que entre el medio y basal (Fig. 9). Borde anterior de los dientes truncados en machos y agudos en hembras (Fig. 10). Espolón de tibia anterior subcilíndrica y ápice truncado, con ligera curvatura en la base (similar en ambos sexos) (Figs. 9 y 10). Fémur medio con suave marginación. Tibia media con pequeñas denticiones en el borde externo. Tarso medio con basitarso subtriangular, una vez y media menor que el segundo; tarsómeros 2-4 subcuadrados, 5 cilíndrico, uñas sin dentición basal (Fig. 11). Tarsos posteriores similares en forma a los medios, uñas con leve dentición en la base (Fig. 12). Abdomen. Esternitos lisos, 4 una vez y media más grande que los demás. En las hembras el 5 y 6 son 0.1 más grandes que en los machos. Pigidio subtriangular sin quilla que separa el propigidio, liso y con marginación apical evidente (Fig. 13).

Órgano genital masculino. Edéago: parámeros subrectangulares y asimétricos (Fig. 14). Saco interno con lóbulo en región submedial y pequeño cepillo (Fig. 15). Esclerito basal de forma circular, con mango subrecto y anillo con borde delgado (Fig. 16). Esclerito placa subcuadrado, con borde inferior redondeado (Fig. 17), esclerito elongado con base irregular, proyección filamentosa hacia el borde inferior, pequeño esclerito suelto en el margen interno (Fig. 18), segmento genital con bordes laterales esclerotizados subtriangulares y paralelos entre sí, escleritos centrales filiformes y no se tocan (Fig. 19).

Alotipo hembra. Difiere del macho en los dientes apicales de la tibia anterior que son más angulados (puede variar por el desgaste del ejemplar) y los esternitos 5 y 6 más largos. Espermateca en forma de media luna, con ángulo amplio y en el borde basal con 2 proyecciones (Fig. 20).

Variación. La longitud total varía entre 7 y $9 \mathrm{~mm}$. Todos los ejemplares examinados son similares en todas las estructuras.

Etimología. El nombre de la especie se refiere a la localidad tipo, el municipio de Arcabuco, Departamento de Boyacá, Colombia.

Material examinado. Holotipo y alotipo. COLOMBIA, Boyacá, municipio de Arcabuco, Pómeca, 548'80"N 73²8'97'O. 2650 m., abril 1997, trampa con excremento humano. Col. F. Escobar (Holotipo: IAVH-E 110160, Alotipo: IAVH-E 110161). 16 paratipos: 3h y $1 \mathrm{~m}$ (CECC) y 11 h., 1 m. (IAVH). Otro material: COLOMBIA, Boyacá, municipio de Arcabuco, vereda Peñas Blancas, 5046'52'N $73^{\circ} 26^{\prime} 45^{\prime \prime} \mathrm{O}, 2546 \mathrm{~m}$., I-VI/2007, trampa excremento humano, Col. J. A. Arias. 23 ejemplares (UPTC).

Hábitat. Arias (2008) recolectó esta especie en bosques de roble, en un paisaje fragmentado, con pocas capturas y especialmente en los fragmentos de mayor tamaño. La mayoría de las especies del género se han recolectado en alturas menores de $500 \mathrm{~m} ; S$. balachowskyi se recolectó a 1 $200 \mathrm{~m}$ en las montañas de Aragua, Venezuela (Martínez y Halffter, 1972) y S. darlingtoni, a 1600 m en las montañas de la sierra nevada de Santa Marta (Noriega et al., 2007). En alturas mayores a $1000 \mathrm{~m}$ se han recolectado $S$. kastneri en Ecuador (Celi et al., 2004) y S. nigriceps en Brasil (Medina, 2002). Scybalocanthon arcabuquensis sp. n. es el primer registro del género a más de $2000 \mathrm{~m}$ de elevación y es la especie del género que se ha recolectado a mayor altura.

\section{Comentarios taxonómicos}

Para separar Scybalocanthon de Canthon, Martínez (1948, 1949) hace énfasis en 3 estructuras principales: el basitarso de las patas medias y posteriores notablemente más corto que el segundo; esta característica quedaba ambigua respecto a si al menos era 2 veces más corto o sólo un poco. En la mayoría de las especies estudiadas se pudo observar que el tamaño de este artejo respecto al segundo es al menos 2 veces más corto, y sólo en $S$. arcabuquensis (Fig. 11) y S. aff. nigellus (Figs. 27 y 38) es al menos una vez y media (Fig. 21-42).

El género presenta variación en el margen basal del pigidio; a pesar de la corrección que realizó Martínez (1949) proponiendo que está ausente, en las especies estudiadas en este trabajo (Fig. 43-53) se observó que en la mayoría está presente de manera muy tenue. Sólo en $S$. aff. pygidialis 2 está ausente (Fig. 50) y en $S$. aff. nigellus está levemente marcado (Fig. 49). Hipómero (Figs. 113-125). Esta estructura llamada proepisterno por Martínez (1949); él describe que en las especies de Scybalocanthon no hay carina transversal, ni dentículo en el borde lateral. Se observa que algunas de las especies estudiadas no poseen carina transversal (Figs. 114, 115, 116, 117, 120, 122, 123, 124, 125), pero otras, como $S$. arcabuquensis sp. n. (Fig. 121), S. moniliatus (Fig. 118), S. maculatus (Fig. 123) у S. trimaculatus (Fig. 125), tienen una pequeña carina en el borde interno, un poco saliente en algunos casos, como S. aereus (Fig. 119) y S. aff. nigellus (Fig. 113). Todas las especies de Scybalocanthon carecen del dentículo en el borde lateral del pronoto. 
Órgano genital masculino de las especies de Scybalocanthon

Edéago (Figs. 54-68). La estructura del edéago ha sido ampliamente usada en Canthon para la separación de especies (Halffer, 1961; Halffter y Martínez, 1977; Medina et al., 2003). Estos autores han descrito la forma, encontrando diferentes tipos de edéago en cada uno de los grupos estudiados. Una de las características evidentes es la simetría de los parámeros. En la mayoría de los cantoninos americanos, los parámeros son simétricos (Medina y Molano, datos no publicados). En las especies estudiadas de Scybalocanthon los parámeros son asimétricos, con excepción de $S$. aereus (Fig. 67). Otros Canthonini con parámeros asimétricos son Canthon aberrans (Medina et al., 2003), Sylvicanthon bridarollii y Streblopus opatroides (C. A.Medina, datos no publicados). La forma de los parámeros es un carácter muy variable en las especies de Scarabaeinae. Medina et al. (2003) describen 4 tipos para las especies de Canthon (triangular, rectangular, forma de bota, y estrecha hacia el ápice y cara ventral sinuada). En las especies de Scybalocanthon, se evidencian los 2 primeros tipos: la mayoría de las especies presentan una forma rectangular, generalmente con pequeña proyección en la cara dorsal y ventral. Como caso especial está $S$. trimaculatus, con parámeros triangulares (Fig. 60) y $S$. aff. pygidialis 1 (Fig. 58), en que el parámero izquierdo tiene el ápice con 2 puntas una dorsal y otra ventral. Saco interno del edéago (Figs. 69-79). Las especies de Scybalocanthon presentan en la región submedial un lóbulo corto que generalmente está cubierto de pequeñas espinas o escamas, que en $S$. trimaculatus se observa reducido (Fig. 73). Este lóbulo ha sido observado en otros Canthonini como en Canthon bicolor (Medina et al., 2003) y en las especies de Deltochilum (Deltohyboma) que es más largo (Molano et al., datos no publicados). En la mayoría de las especies de Scybalocanthon se encuentra una estructura semiovalada, formada por dientes grandes, en este artículo denominada "cepillo", muy común en las especies de Canthon (Medina et al., 2003). Escleritos apicales. En Scybalocanthon se observan los 3 tipos de escleritos estudiados por Medina et al. (2003) en Canthon. El esclerito basal (Figs. 80-90) es circular, con un anillo grande, de bordes delgados y un mango estrecho sin proyección basal, con ápice ensanchado y pequeña curvatura entre éste y el anillo. El esclerito placa en este género se encuentra en posición opuesta al esclerito basal; la forma de esta estructura en las especies de Scybalocanthon estudiadas varía significativamente (Figs. 91-101). Sin embargo, se presentan patrones por grupo de especies: la forma rectangular con ápices estrechos y esclerotizado es característica de $S$. maculatus (Fig. 91), S. darlingtoni (Fig. 96), S. kelleri (Fig. 98), S. moniliatus (Fig. 99), S. aereus (Fig. 100) y S. arcabuquensis sp. n.
(Fig. 17). El otro tipo de esclerito es membranoso y varía en forma, se encuentra en S. nigriceps (Fig. 92), S. aff. pygidialis 1 y 2 (Figs. 93 y 94), S. trimaculatus (Fig. 95), S. aff. pygidialis 3 (Fig. 101) y ausente en $S$. aff. pygidialis 4 (Fig. 97). En algunas especies se encuentra una serie de dientes en forma de cepillos en esta área: $S$. aff. pygidialis 2 (Fig.94), S. aff. pygidialis 4 (Fig. 97) y S. aff. pygidialis 3 (Fig. 101). En las especies de Scybalocanthon, el esclerito elongado se encuentra ubicado entre los escleritos circular y placa. Es una estructura de forma muy similar en todas las especies (Figs. 102-112), con una parte basal ensanchada a manera de placa triangular, y superpuesta con otra un poco irregular, y un cuerpo formado por una estructura elongada de grosor diferente y siempre terminando en un filamento, ya sea corto o largo, expuesto o no. El esclerito elongado puede estar acompañado por un pequeño esclerito lateral, o 2 como en $S$. trimaculatus son 2 (Fig. 106).

Los géneros Canthon y Scybalocanthon están estrechamente relacionados. Tradicionalmente ha habido dificultad en la separación de las especies de estos dos géneros que presentan similitudes en su morfología, además comparten los mismos tipos de hábitats y en algunas especies su asociación con el excremento de monos. Usando la morfología interna del órgano genital de los machos se ha podido separar y definir grupos naturales de especies, i.e. el género africano Epirinus (Medina y Scholtz, 2005), Deltochilum (Gonzáles et al., 2009) y otros en revisión como Uroxys (Escobar y Medina, datos no publicados.). En el caso de Canthon y Scybalocanthon se observan diferencias claras en la forma del saco interno como el lóbulo lateral desarrollado; la forma de los escleritos, en especial el basal, con un anillo muy amplio y cepillos en el lóbulo lateral y la región apical. Estos caracteres han podido ser confrontados con caracteres de la morfología externa: el primer tarsómero de las patas medias y posteriores relativamente más corto que el segundo y subrectangular; margen basal que separa el propigidio del pigidio ausente o poco marcado; e hipómero sin carina transversa o muy corta, sin dentículo en la región externa. Estos caracteres facilitan la separación clara de estos dos géneros.

\section{Agradecimientos}

A las colecciones biológicas del Instituto A. V. Humboldt por facilitar el material de estudio; a Alejandro Lopera y a la Colección Entomológica, Museo de Historia Natural "Luis Gonzalo Andrade", Universidad Pedagógica y Tecnológica de Colombia, Tunja, Boyacá, Colombia (UPTC); de manera especial a Jorge A. Arias, 
por las colectas de material más reciente; a Fabio Arturo González, por la ayuda en la edición de esquemas y la disección de los órganos genitales; a Roberto Rozo, por la ilustración del hábitus, y a los evaluadores anónimos que enriquecieron este escrito.

\section{Literatura citada}

Arias, J. A. 2008. Estructura de escarabajos coprófagos (Scarabaeidae: Scarabaeinae) en tres paisajes fragmentados en Arcabuco, Boyacá. Tesis, Universidad Pedagógica y Tecnológica de Colombia. 82 p.

Celi, J., E. Terneus, J. Torres y M. Ortega. 2004. Dung beetles (Coleoptera: Scarabaeinae): Diversity in an altitudinal gradient in the Cutucú Range, Morona Santiago, Ecuador Amazon. Lyonia 7:38-52.

González, F. A., F. Molano y C. A. Medina. 2009. Los subgéneros Calhyboma Kolbe 1893, Hybomidium Shipp 1897 y Telhyboma Kolbe 1893 de Deltochilum (Coleoptera: Scarabaeidae: Scarabaeinae) en Colombia. Revista Colombiana de Entomología 35:253-274.

Halffter, G. 1961. Monografía de las especies norteamericanas del género Canthon Hoffsg. (Coleoptera, Scarabaeidae). Revista Hispanoamericana de Ciencias Puras y Aplicadas 20:225-320.

Halffter, G. y Martínez A. 1977. Revisión monográfica de los Canthonina americanos IV: Clave para géneros y subgéneros. Folia Entomológica Mexicana 38:29-107.

Howden, H. y O. P. Young. 1981. Panamanian Scarabaeinae taxonomy, distribution and habits (Coleoptera: Scarabaeidae). Contributions of the American Entomological Institute 18:1-204.

Martínez, A. 1948. Insectos nuevos o poco conocidos IV (Col. Scarabaeidae). Revista de la Sociedad Entomológica Argentina 14:3-11.

Martínez, A. 1949. Insectos nuevos o poco conocidos VII. Revista de la Sociedad Entomológica Argentina 14:175-193.

Martínez A. y Halffter G. 1972. New taxa of American Canthonina. Entomologische Arbeiten aus dem Museum G. Frey 23:33-66.

Medina, M.I. 2002. The night and day of dung beetles (Coleoptera, Scarabaeidae) in the Serra do Japi, Brasil: Elytra colour related to daily activity. Revista Brasileira de Entomologia 46:597-600.

Medina, C. A., A. Lopera, A. Vitolo y B. Gill. 2001. Escarabajos coprófagos (Coleoptera, Scarabaidae: Scarabaeinae) de Colombia. Biota Colombiana 2:131-144.

Medina, C. A., C. Scholtz y B. D. Gill. 2003. Morphological variation and systematics of Canthon Hoffmannsegg 1817, and related genera of New World Canthonini dung beetles (Coleoptera, Scarabaeidae). Deutsche Entomologische Zeitschrift 50:23-68.

Medina, C. A. y C. Scholtz. 2005. Systematics of the southern African genus Epirinus Reiche (Coleoptera: Scarabaeinae: Canthonini): descriptions of new species and phylogeny. Insect Systematics and Evolution 36:145-160.

Noriega, J. A., C. Solis, F. Escobar y E. Realpe. 2007. Escarabajos coprófagos (Coleoptera: Scarabaeidae) de la provincia de la Sierra Nevada de Santa Marta. Biota Colombiana 8:77-86.

Pereira, F.S. y A. Martínez. 1956. Os generos de Canthonini americanos (Col. Scarabaeidae). Revista Brasileira de Entomologia 6:91-192. 


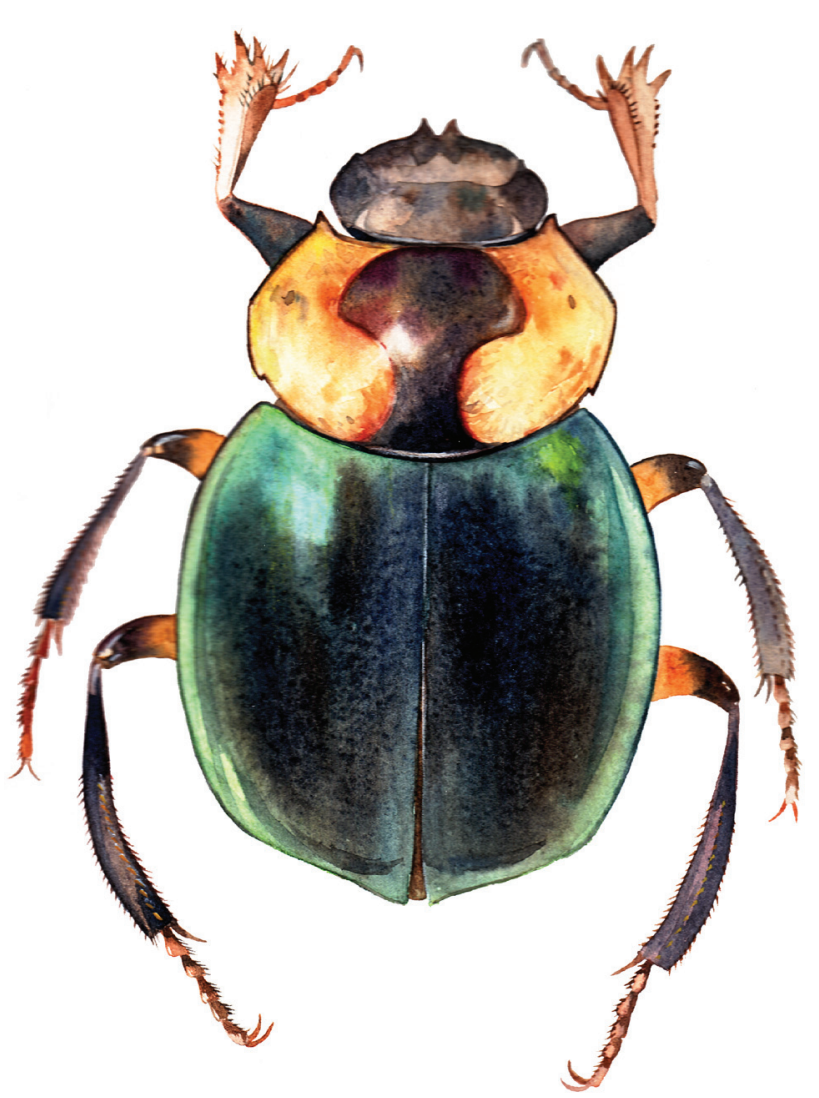

Figura 1. Habitus de Scybalocanthon arcabuquensis n. sp.


Figuras 7-12. Tórax de Scybalocanthon arcabuquensis n. sp. 7, pronoto vista dorsal; 8 , hipómero; 9, tibia anterior macho; 10 , ápice de la tibia anterior hembra; 11, pata media; 12 , pata posterior.

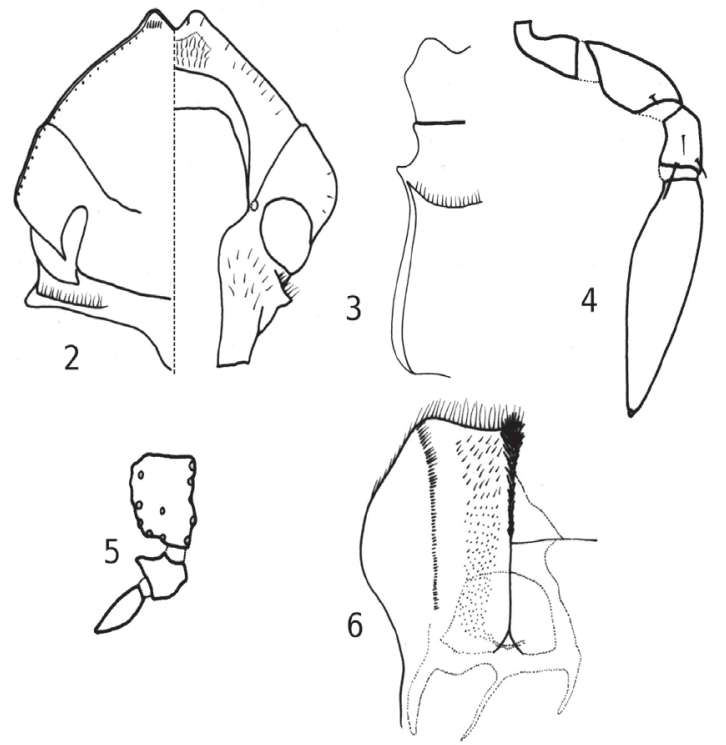

Figuras 2-6. Cabeza de Scybalocanthon arcabuquensis n. sp. 2, vista dorsal (izquierda) y vista ventral (derecha); 3, labio; 4, palpo maxilar; 5, palpo labial; 6, epifaringe.

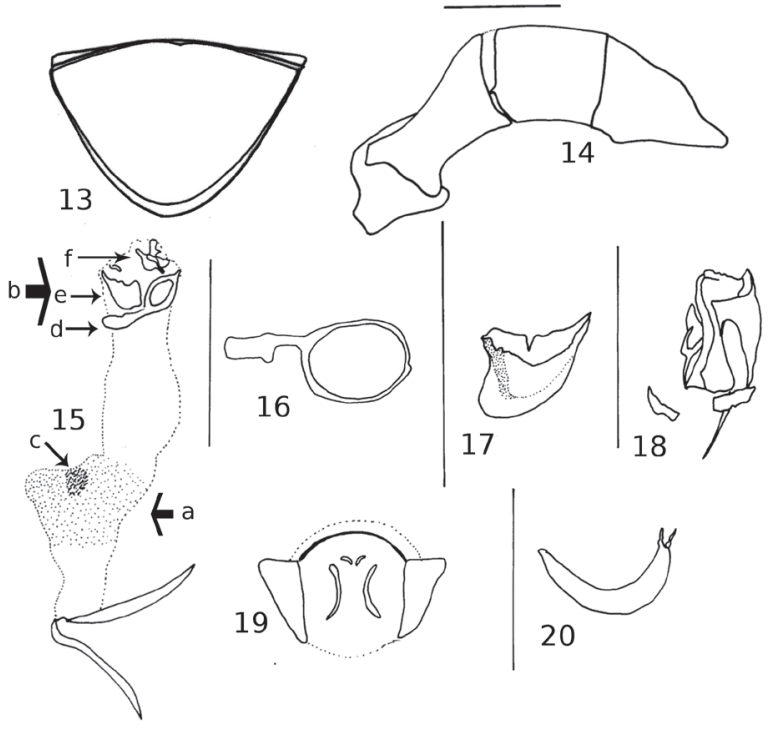

Figuras 13-20. Abdomen y aparato reproductor de Scybalocanthon arcabuquensis n. sp. 13, pigidio; 14, edéago; 15, saco interno: a) región submedial, b) región apical, c) cepillo del lobulo, d) esclerito basal, e) esclerito placa, f) esclerito elongado; 16, esclerito basal; 17 , esclerito placa; 18 , esclerito elongado; 19 , segmento genital; 20, espermateca. Escala $1 \mathrm{~mm}$. 

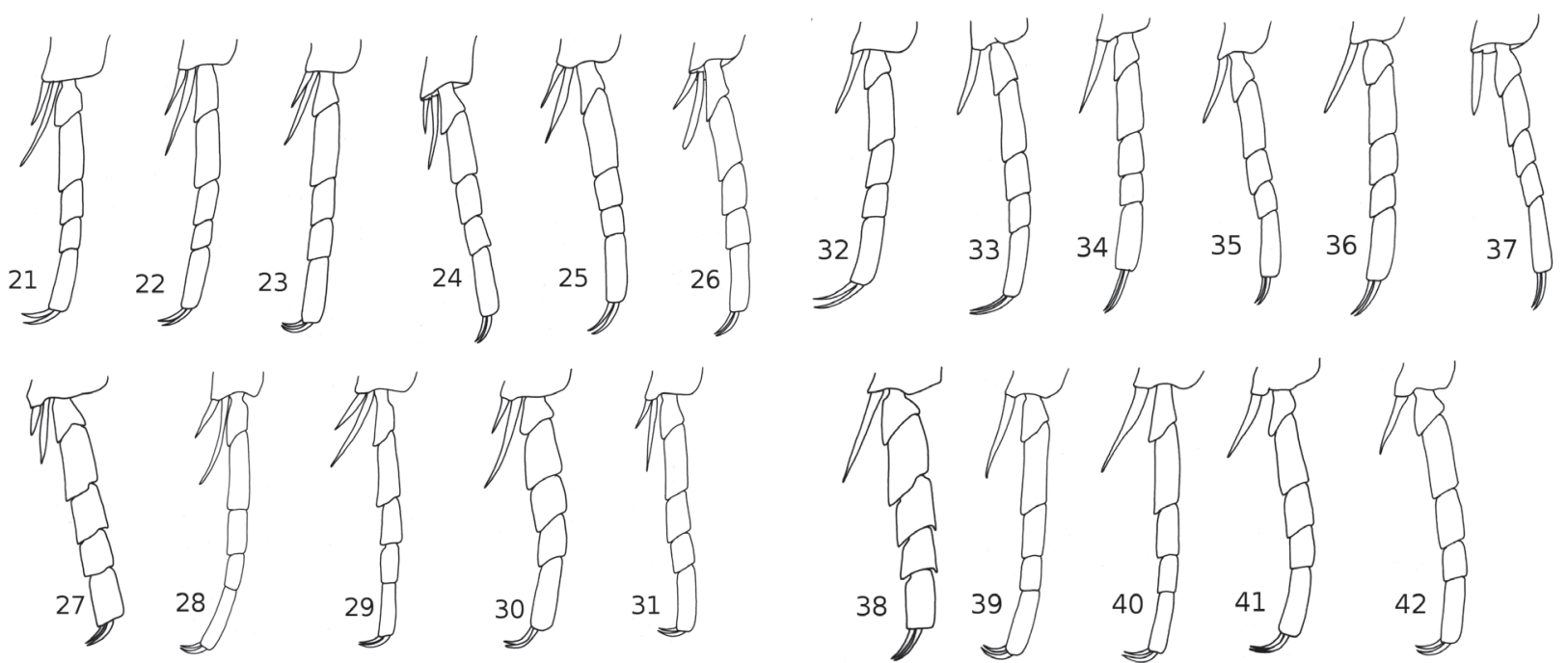

Figuras 21-31. Tarsos de las patas medias de Scybalocanthon. 21, S. darlingtoni, 22, S. aff. pygidialis 4; 23, S. kelleri, 24, S. maculatus, 25, S. moniliatus, 26, S. nigriceps, 27, S. aff. nigellus, 28, S. aff. pygidialis $1 ; 29$, S. aereus, 30, S. aff. pygidialis $3 ; 31$, S. trimaculatus.

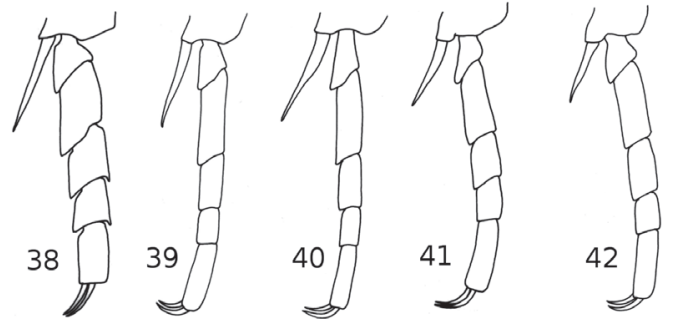

Figuras 32-42. Tarsos de las patas posteriores de Scybalocanthon. 32, S. darlingtoni; 33. S. aff. pygidialis $4 ; 34$, S. kelleri, 35, S. maculatus, 36, S. moniliatus, 37, S. nigriceps, 38, S. aff. nigellus, 39, S. aff. pygidialis $1 ; 40, S$. aereus, $41, S$. aff. pygidialis $3 ; 42$, S. trimaculatus
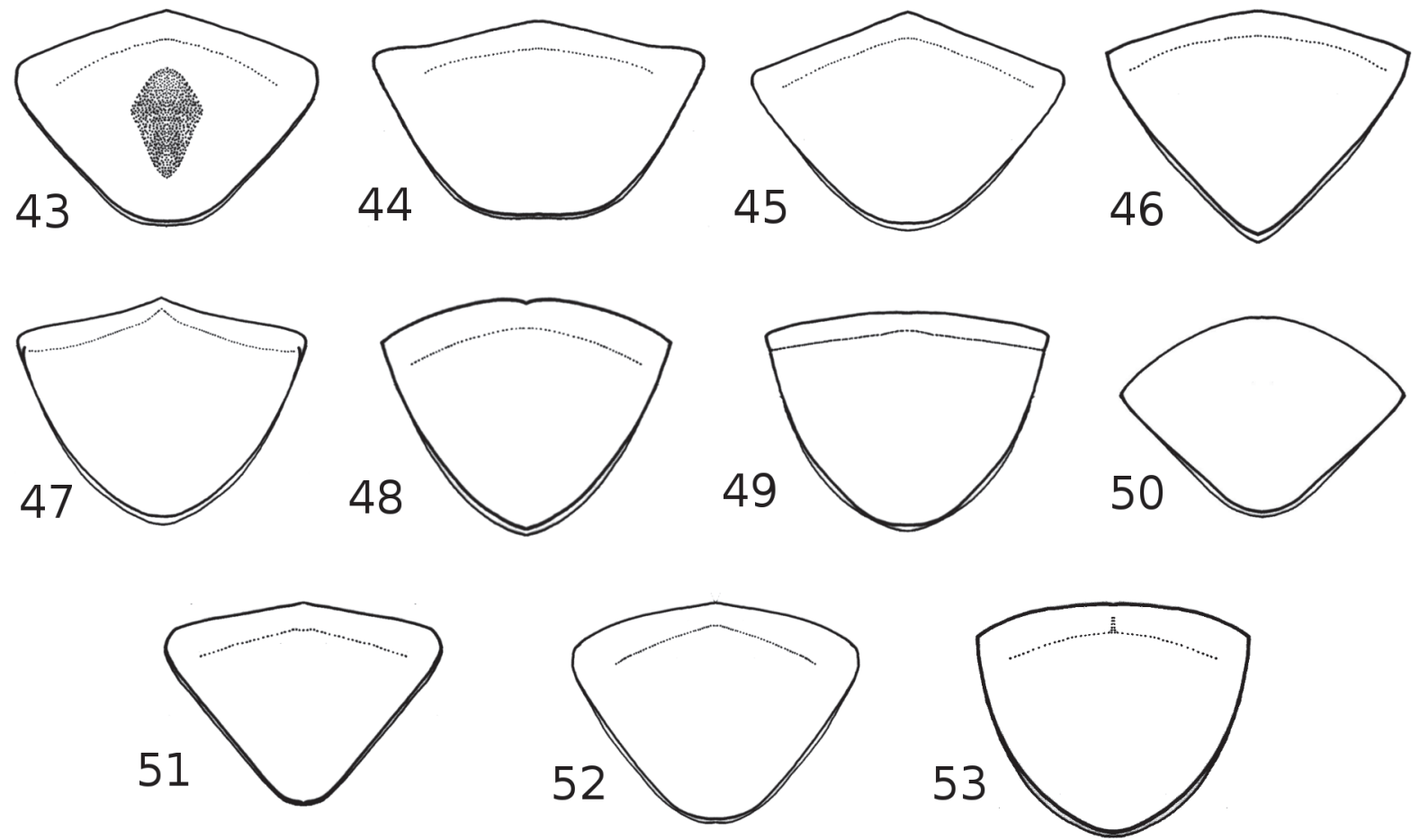

Figuras 43-53. Pigidio de Scybalocanthon. 43, S. darlingtoni; 44, S. aff. pygidialis 4; 45, S. kelleri; 46, S. maculatus; 47, S. moniliatus; 48, S. nigriceps; 49, S. aff. nigellus; 50, S. aff. pygidialis $1 ; 51, S$. aereus; 52, S. aff. pygidialis $3 ; 53$, S. trimaculatus. 

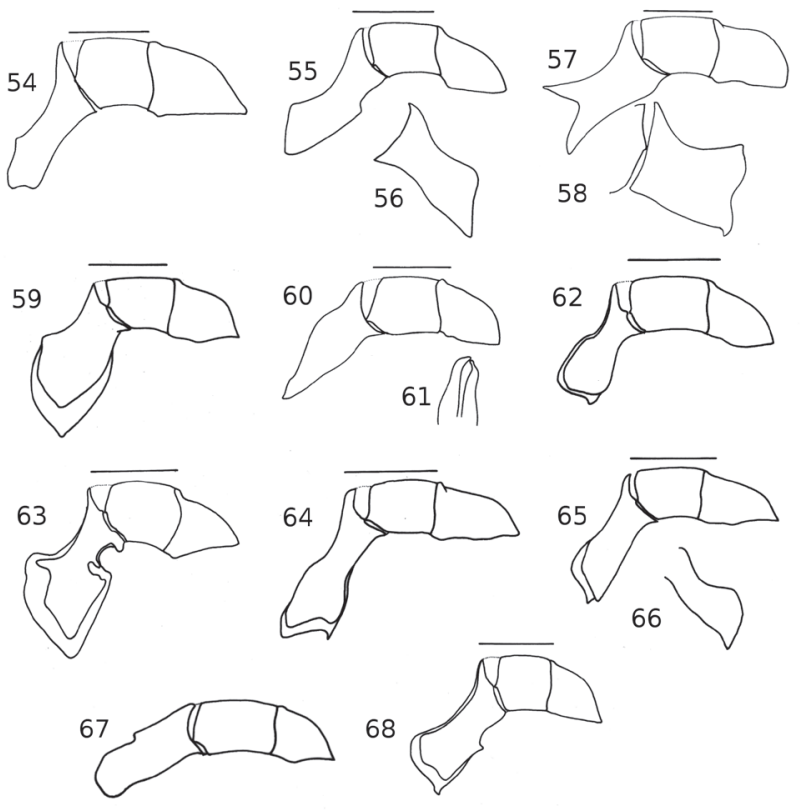

68

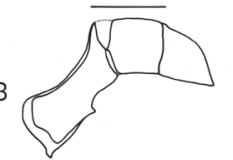

Figuras 54-68. Edéago de Scybalocanthon. 54, S. maculatus; 55, S. nigriceps; 56, parámero derecho de $S$. nigriceps; $57, S$. aff. pygidialis $1 ; 58$, parámero derecho $S$. aff. pygidialis $1 ; 59$, $S$. aff. pygidialis $2 ; 60, S$. trimaculatus; 61 , parámero derecho $S$. trimaculatus; 62, S. darlingtoni; 63, S. aff. pygidialis $4 ; 64, S$. kelleri; 65, S. moniliatus; 66, parámero derecho S. moniliatus; 67, S. aereus; 68, $S$. aff. pygidialis 3.

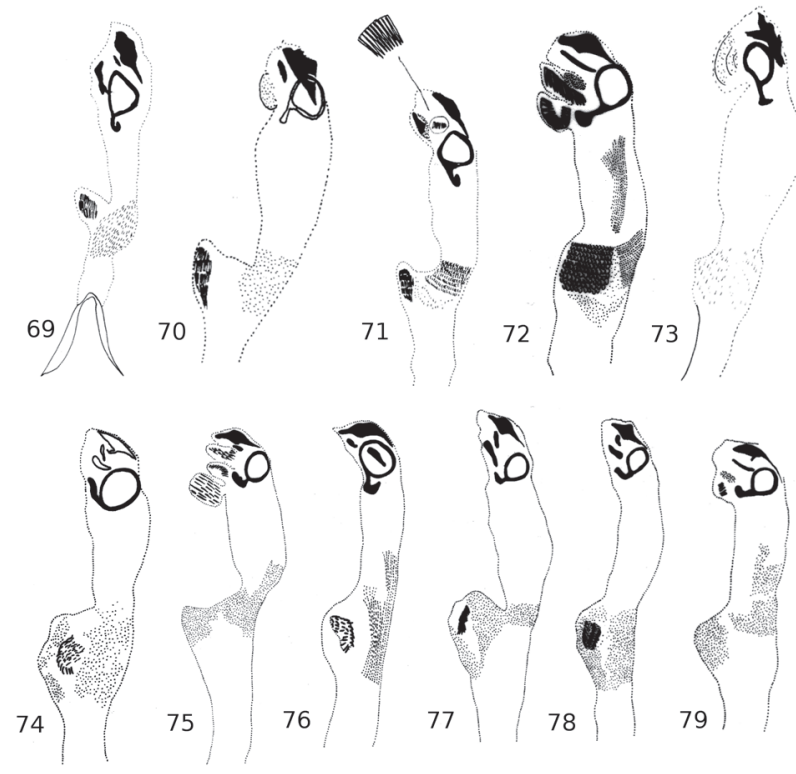

Figuras 69-79. Saco interno de Scybalocanthon. 69. S. maculatus; 70, S. nigriceps, 71, S. aff. pygidialis $1 ; 72$. S. aff. pygidialis 2 , 73. S. trimaculatus; 74. S. darlingtoni; 75. S. aff. pygidialis $4 ; 76$. S. kelleri; 77. S. moniliatus; 78. S. aereus; 79. S. aff. pygidialis 3.

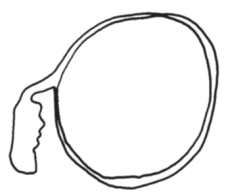

80

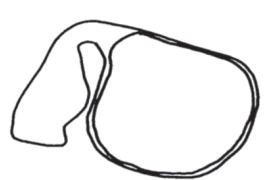

84

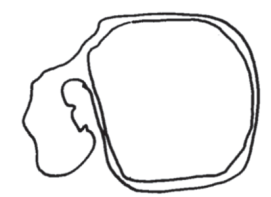

81

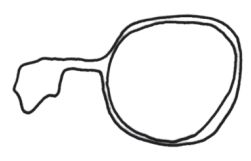

85

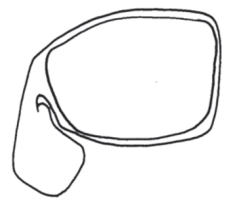

82

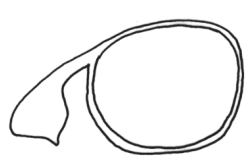

86

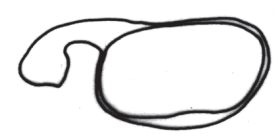

83

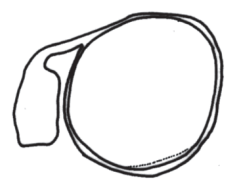

87

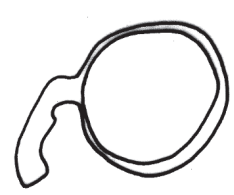

88

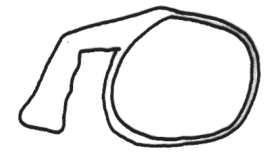

89

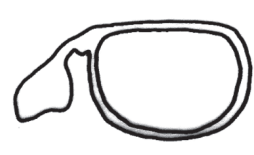

90

Figuras 80-90. Esclerito basal de Scybalocanthon. 80, S. maculatus; 81, S. nigriceps; 82, S. aff. pygidialis 1; 83, S. aff. pygidialis 2; 84, S. trimaculatus; 85, S. darlingtoni; 86, S. aff. pygidialis 4; 87, S. kelleri; 88, S. moniliatus; 89, S. aereus; 90, S. aff. pygidialis 3. 

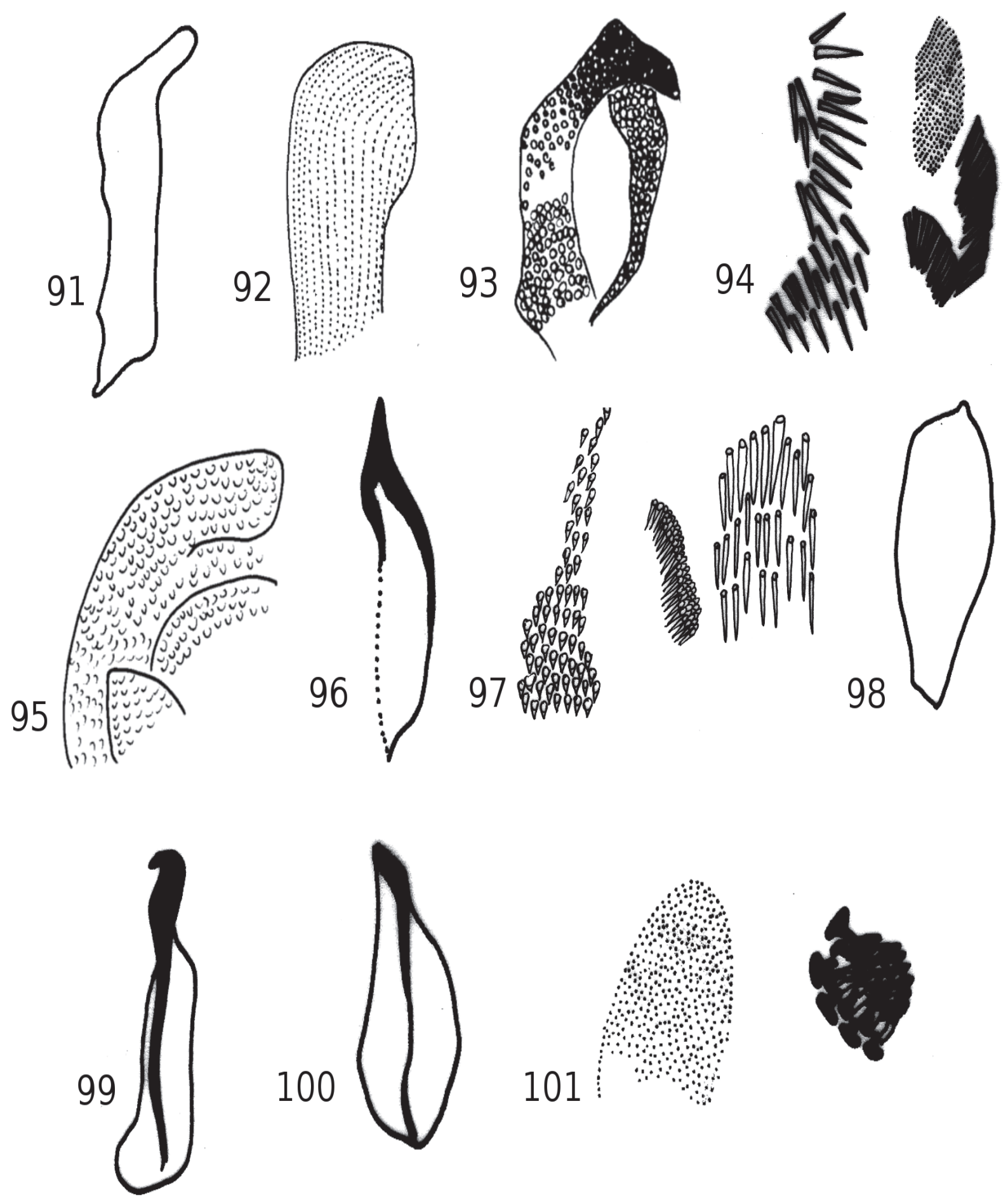

Figuras 91-101. Esclerito placa de Scybalocanthon. 91, S. maculatus; 92, S. nigriceps; 93, S. aff. pygidialis 1; 94, S. aff. pygidialis 2; 95, S. trimaculatus; 96, S. darlingtoni; 97, S. aff. pygidialis 4; 98, S. kelleri; 99, S. moniliatus; 101, S. aereus; 101, S. aff. pygidialis 3. 

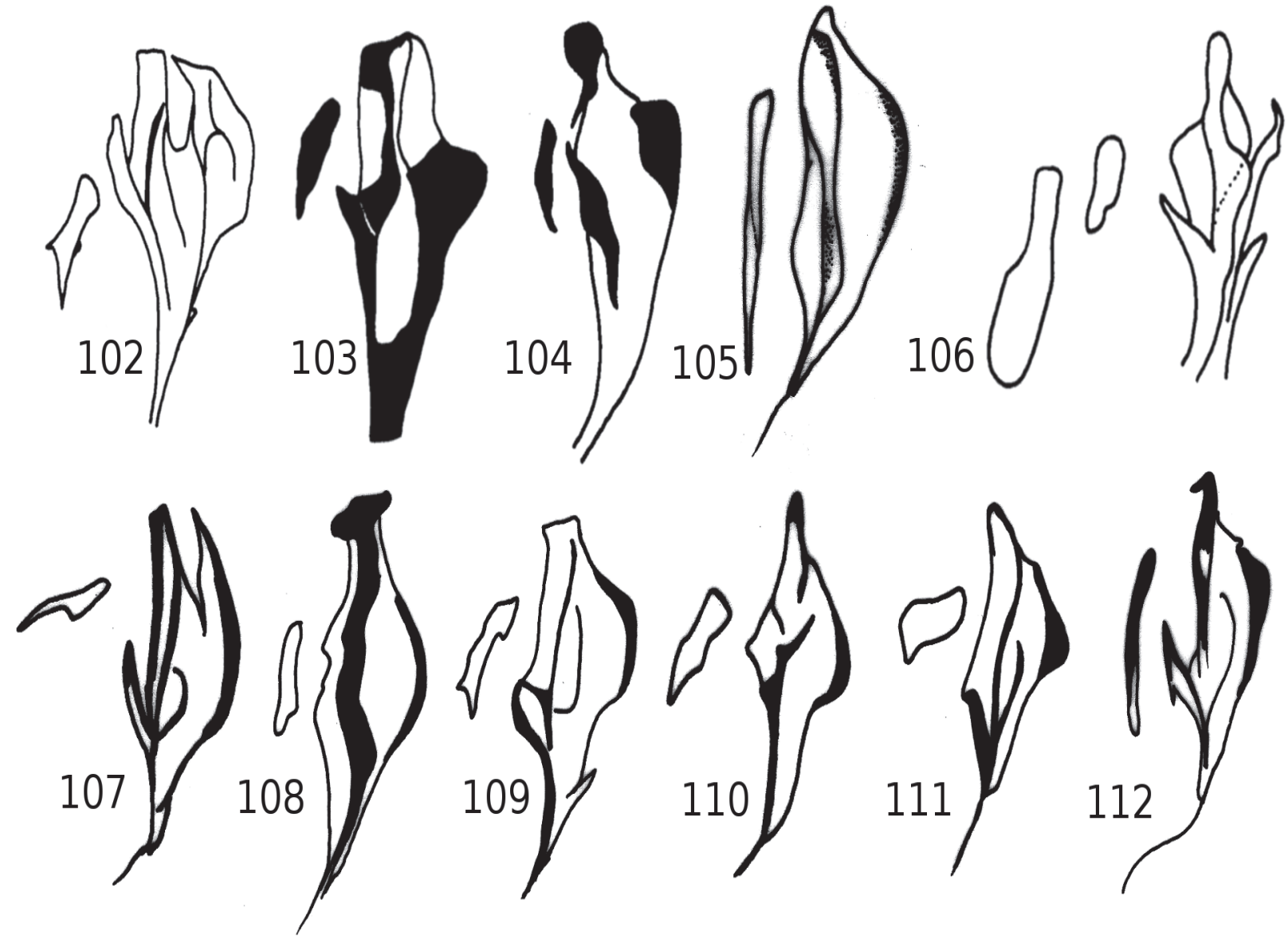

Figuras 102-112. Esclerito elongado de Scybalocanthon. 102, S. maculatus; 103, S. nigriceps; 104, S. aff. pygidialis 1; 105, S. aff. pygidialis 2; 106, S. trimaculatus; 107, S. darlingtoni; 108, S. aff. pygidialis 4; 109, S. kelleri; 110, S. moniliatus; 111, S. aereus; 112, S. aff. pygidialis 3 . 

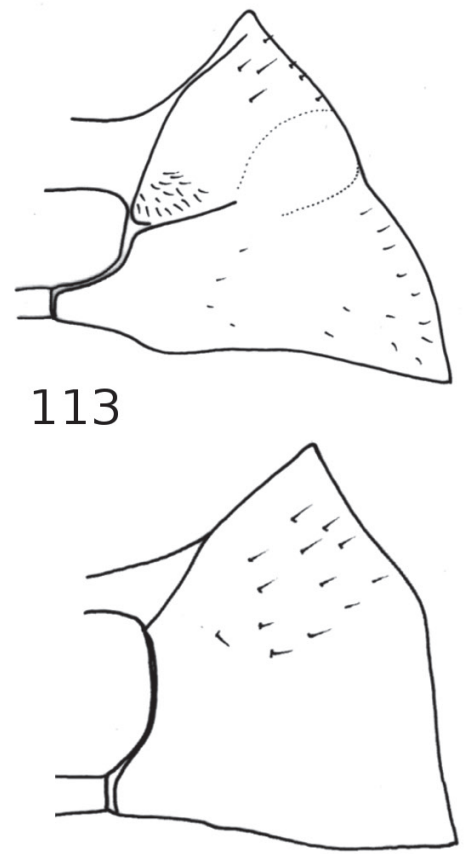

116

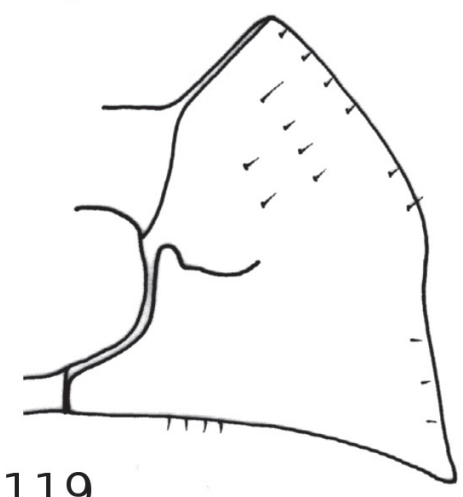

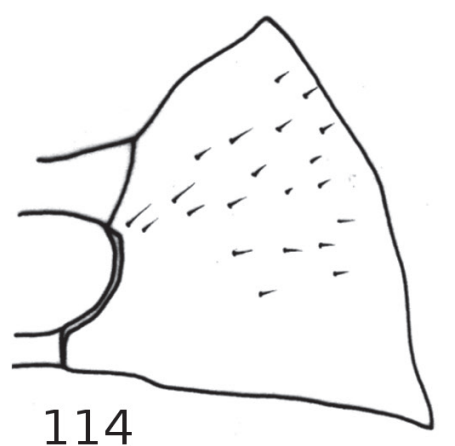

114



117

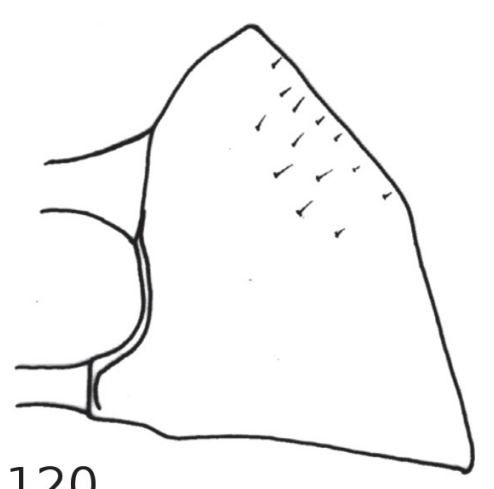

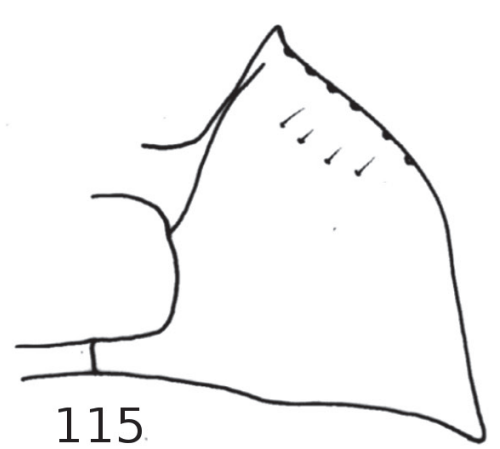
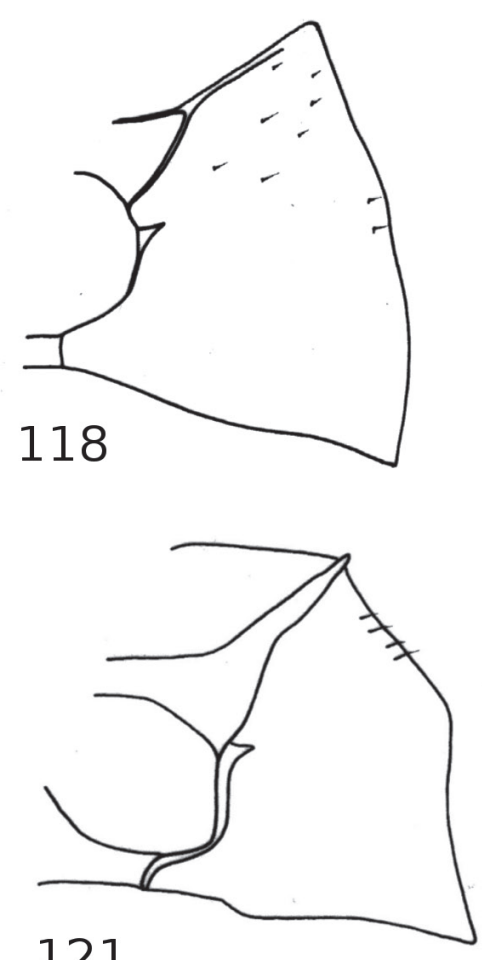

121

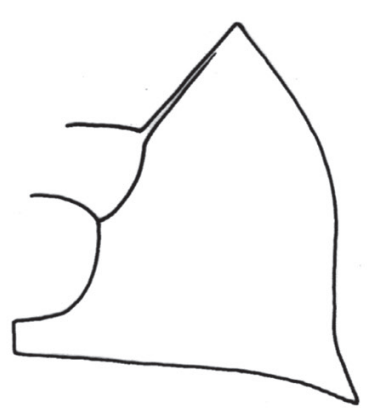

122

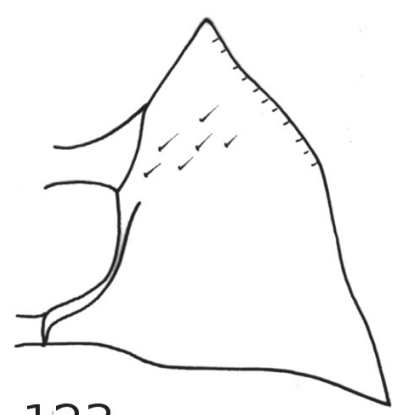

123

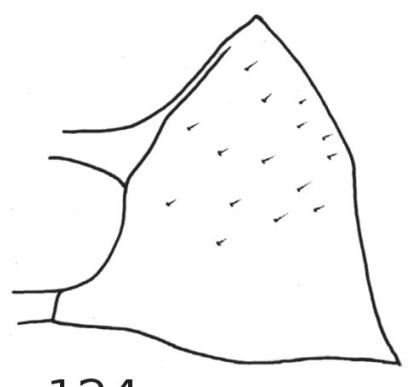

124

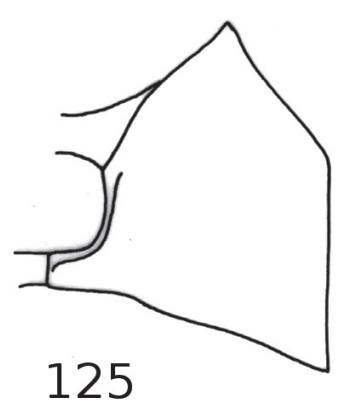

Figuras 113-125. Hipómero de Scybalocanthon. 113, S. aff. nigellus; 114, S. aff. pygidialis 2; 115, S. darlingtoni; 116, S. aff. pygidialis 4; 117, S. keller; 118, S. moniliatus; 119, S. aereus; 120, S. aff. pygidialis 3; 121, S. arcabuquensis sp. nov.; 122, S. aff. pygidialis 1; 123, S. maculatus; 124, S. nigriceps; 125, S. trimaculatus. 
